Pacific Journal of Mathematics

S-SPACES AND THE OPEN MAPPING THEOREM 


\title{
$S$-SPACES AND THE OPEN MAPPING THEOREM
}

\author{
TAQDIR HUSAIN
}

1. Introduction. Let $E$ be a locally convex Hausdorff topological vector space over the field of real numbers and $E^{\prime}$ its dual. Let $t_{p}$ denote the uniform convergence topology over precompact sets of $E$ on $E^{\prime}$. It is known ([2], Chapitre III, $\S 3, \mathrm{~N}^{\circ} .5$, Proposition 5) that $t_{p}$ topology coincides with the weak ${ }^{*}$ topology $\sigma\left(E^{\prime}, E\right)$ on each equicontinuous set of $E^{\prime}$. Let $t_{w}$ denote the finest locally convex topology on $E^{\prime}$ which coincides with $\sigma\left(E^{\prime}, E\right)$ on each equicontinuous set. Following H. S. Collins [3] let $e-w^{*}$ denote the finest topology which coincides with $\sigma\left(E^{\prime}, E\right)$ on each equicontinuous set of $E^{\prime}$. It is clear that these three topologies are related as follows: $e-w^{*} \supset t_{w} \supset t_{p}$ or, in other words, $e-w^{*}$ is finer than $t_{w}$ and $t_{w}$ is finer than $t_{p}$. Collins [3] has shown that in general these inclusions are proper. However, if $E$ is metrizable, $e-w^{*}=t_{p}$.

The object of this paper is to study the locally convex linear spaces $E$ on whose dual $E^{\prime}, e-w^{*}=t_{p}$. Such a space we call an $S$-space. We shall give examples showing that an $S$-space is a proper generalization of metrizable locally convex linear spaces. The completion of an $S$-space is an $S$-space. A complete $S$-space is $B$-complete (a notion due to V. Pták [19] which is important in connection with the open mapping theorem). The Krein Smulian Theorem is true on complete $S$-spaces (Theorem 3.) Two of the main theorems are the following ones:

THEOREM 8. A complete l.c. space with a countable fundamental system of precompact sets is a complete S-space and hence a fortiori B-complete.

THEOREM 10. Let $E$ be a complete S-space in which the closure of any dense subspace is obtained by taking the closures of its precompact sets only. Then $E^{\prime}$, endowed with the $t_{c}$-topology (the uniform convergence topology over convex compact sets of $E$ on $\left.E^{\prime}\right)$ is $B_{r}$-complete for all locally convex topologies finer than $t_{c}$ and coarser than $\tau\left(E^{\prime}, E\right)$.

Theorem 10 is a generalization of result 6.5 [19] concerning Fréchet spaces. We also prove that an $L F$-space $E$ with a defining sequence of Fréchet spaces $E_{n}(n \geqq 1)$ is $B$-complete provided each $E_{n}$ contains a

Received February 24, 1961. Presented to the Society, October 22, 1960. This work was done at Syracuse University and represents the major part of a thesis submitted to Syracuse University September, 1960, in partial fulfillment of the requirements for the Ph.D. degree. Part of this work was sponsored by the Office of Ordnance Research, U. S. Army. 
countable fundamental system of precompact sets. In general the fact that an $L F$-space need not be $B$-complete follows from an example given by $\mathrm{A}$. Grothendieck [8]. In connection with the open mapping theorem we prove the following: Let $E$ be an $F$-space (or a complete $S$-space) and $F$ a $T$-space (or a $t$-space). Then a linear and continuous mapping of $E$ onto $F$ is open. For other generalizations of Banach's open mapping theorem see Dieudonné et Schwartz [6], Grothendieck [9], Köthe [15], Pták [19] and Robertsons [21].

The author wishes to express his sincere appreciation to professor Mark Mahowald for his assistance and for his many instructive conversations. His thanks are also due to Professor $\mathrm{W}_{r}$. B. Jurkat for his helpful suggestions.

2. Preliminaries and notations. We shall usually follow Bourbaki definitions and notations [2]. The phrases "a topological vector space" or "a locally convex topological vector space" will be abbreviated to "a TVS" or "an l.c. space." A TVS which is metrizable and complete is called an $F$-space $([1], \S 1$, p. 35) and an l.c. $F$-space a Fréchet space ([2], Chap. 2, p. 59). The strict inductive limit of Fréchet spaces is called an $L F$-space [6].

Let $e-w^{*}$ denote the finest topology on the dual $E^{\prime}$ of an l.c. space $E$, which coincides with the weak* topology $\sigma\left(E^{\prime}, E\right)$ on each equicontinuous set. This topology is not necessarily locally convex or even linear. However, by Collins' Theorem 5 [3] it is semi-linear (i.e., the mappings: $(x, y) \rightarrow x-y$ and $(\lambda, x) \rightarrow \lambda x$ are continuous in each variable separately, unlike the linear topology in which these mappings are continuous in both variables together. $e-w^{*}$ can be described more explicitly as follows: A set $W^{\prime}$ of $E^{\prime}$ is $e-w^{*}$-open (or $e-w^{*}$-closed) if and only if $W^{\prime} \cap U^{\circ}$ ( $U^{\circ}$ is the polar of $U$ ) is open (or compact) in the relative weak* topology of $U^{\circ}$ for each neighborhood $U$ of 0 in $E$. A linear mapping $f$ of an l.c. space $E$ into another l.c. space $F$ is said to be almost open if for each neighborhood $U$ of a point $x \in E, \overline{f(U)}$ (the closure of $f(U)$ ) is a neighborhood of the point $f(x) \in F$ [19]. An 1.c. space $E$ is said to be $B$-complete if for an 1.c. space $F$, a linear, continuous and almost open mapping of $E$ into $F$ is open [19]. A set $Q^{\prime}$ in $E^{\prime}$ is said to be almost closed if $Q^{\prime} \cap U^{\circ}$ is weak* closed for each neighborhood $U$ of 0 in $E$. In terms of the $e-w^{*}$-topology to say that $Q^{\prime}$ is almost closed in $E^{\prime}$ is equivalent to saying that $Q^{\prime}$ is $e-w^{*}$-closed. An l.c. space $E$ is called fully-complete if every almost closed subspace $Q^{\prime}$ of $E^{\prime}$ is weakly closed. Pták [19] proved that the notions of $B$-completeness and full-completeness are equivalent. We shall use the term " $B$-complete" only in the sequel. An. l.c. space $E$ is called $B_{r}$-complete if every almost closed dense subspace $Q^{\prime}$ of $E^{\prime}$ coin- 
cides with $E^{\prime} . B_{r}$-completeness can be described in terms of topologies on $E$. For this let $u$ and $v$ be two locally convex topologies on $E$ such that $u \supset v$. Let $v(u)$ denote the topology for which a fundamental system of neighborhoods of 0 consists of $u$-neighborhoods which are $v$-closed. If $v$ is Hausdorff so is $v(u)$ and $u \supset v(u) \supset v$. Now an l.c. space $E$, endowed with a locally convex topology $u$, is called $B_{r}$-complete if for any locally convex topology $v$ such that $u \supset v$ and $v(u)=v$ imply $u=v$ ([19], 4.2).

Given a family $\subseteq$ of bounded sets of a TVS, then a sub-family $\mathfrak{F}$ is called a fundamental system of $\mathfrak{S}$ if each set of $\mathfrak{S}$ is contained in a set of $\mathfrak{F}[11]$.

The following notations will be used for denoting different topologies: $\sigma\left(E^{\prime}, E\right)=$ the simple convergence topology on $E^{\prime}$ (also called weak*topology)

$t_{c}=$ the uniform convergence topology over convex compact sets of $E$ on $E^{\prime}$.

$t_{k}=$ the uniform convergence topology over compact sets of $E$ on $E^{\prime}$.

$t_{p}=$ the uniform convergence topology over precompact (totally bounded) sets of $E$ on $E^{\prime}$.

$\beta=$ the uniform convergence topology over bounded sets of $E$ on $E^{\prime}$ (also called strong topology).

$\tau\left(E^{\prime}, E\right)=$ the Mackey topology on $E^{\prime}$.

$e-w^{*}=$ the finest topology which coincides with $\sigma\left(E^{\prime}, E\right)$ on each equicontinuous set of $E^{\prime}$.

$t_{w}=$ the finest locally convex topology which coincides with $\sigma\left(E^{\prime}\right.$, $E$ ) on each equicontinuous set of $E^{\prime}$.

There are some similar topologies on an 1.c. space $E$ itself. For example:

$\sigma\left(E, E^{\prime}\right)=$ the weak topology on $E$.

$\tau\left(E, E^{\prime}\right)=$ the Mackey topology on $E$.

$\omega=$ the finest locally convex topology on $E$.

If the dual $E^{\prime}$ of an l.c. space $E$ is endowed with a topology other than $\sigma\left(E^{\prime}, E\right)$, then we shall denote the space by $E^{\prime t_{c}}, E^{\prime t_{p}}, E^{\prime \tau}, E^{\prime \beta}$ etc., depending upon which topology $t_{c}, t_{p}, \tau$ or $\beta$ is put on $E^{\prime}$. A linear space $E$ with a topology $u$ will be denoted by $E_{u}$ and will be assumed Hausdorff over the field of the reals.

The two topologies $u_{1}$ and $u_{2}$ on a linear space $E$ are said to be equivalent if they generate isomorphic duals and the notation for this is $u_{1} \sim u_{2}$. 


\section{S-spaces and their characterization.}

Definition 1. An l.c. space $E_{u}$ is called an $S$-space if on its dual $E^{\prime}, e-w^{*}=t_{p}$.

The definition implies $e-w^{*}=t_{w}$ and consequently $e-w^{*}$ is a locally convex topology for $S$-spaces.

A simple characterization of $S$-spaces is given as follows:

THEOREM 1. An l.c. space $E_{u}$ is an S-space if and only if for each $e$-w $w^{*}$-open neighborhood $W^{\prime}$ of 0 in $E^{\prime}$ there exists a u-precompact set $P$ in $E_{u}$ such that $P^{0} \subset W^{\prime}$.

Proof. If $E_{u}$ is an $S$-space then $e-w^{*}=t_{p}$ on $E^{\prime}$ which implies the condition in the Theorem. On the other hand, if the condition $P^{0} \subset W^{\prime}$ holds, $e-w^{*} \subset t_{p}$. But in general, $e-w^{*} \supset t_{p}$ ([3], Theorem 10) and therefore, by combining the two inequalities, we get $e-w^{*}=t_{p}$ on $E^{\prime}$. In other words, $E_{u}$ is an $S$-space.

Metrizable 1.c. spaces (in particular, Fréchet spaces and Banach spaces) form an important class of $S$-spaces ([3], Theorem 12).

An example of an $S$-space which is not metrizable is as follows:

ExAmPLE. Let $E$ be the linear space of finite sequences (i.e., the sequences with only a finite number of non-zero components), endowed with the finest locally convex topology for which the embeddings: $R e_{n} \rightarrow E$ (where $e_{n}$ is the vector with all components except the $n$ th, zero and $R$ is the field of real numbers) are continuous for $n \geqq 1$. In this case $E$ is the strict inductive limit of an increasing sequence $E_{n}(n \geqq 1)$ of finite-dimensional Euclidean spaces which are metrizable (actually Fréchet spaces). By using the open mapping theorem of Banach and Baire's theorem (Every Fréchet space is of 2nd category), it is easy to see that $E$ is not metrizable. But $E$ is complete. Now we show that $E$ is an $S$-space. For this consider the dual $E^{\prime}=R^{N}$ (the countable product of the reals). Since $E$ is complete, $t_{p}=t_{c}$ on $E^{\prime}$. Moreover, $t_{c}=\sigma\left(E^{\prime}, E\right)$ on $E^{\prime}$ because every convex compact set in $E$ is finite dimensional. But $\sigma\left(E^{\prime}, E\right)$ is simply the product topology which is metrizable and therefore, by Theorem 13 ([13], p. 231), $e-w^{*}=\sigma\left(E^{\prime}, E\right)$. Combining the latter with the above equations we get $e-w^{*}=t_{p}$. This proves that $E$ is an $S$-space.

4. $S$-spaces and $B$-completeness. By one of the examples due to Collins [3] it follows that a complete l.c. space need not be an $S$-space (the example will be quoted later on). Nor is it true that an $S$-space is always complete because a metrizable (not necessarily complete) l.c. 
space is an $S$-space ([3], Theorem 12). The following theorem gives a sufficient condition for an $S$-space to be $B$-complete.

Theorem 2. An S-space $E_{u}$ is B-complete provided every u-closed $u$-precompact set in $E_{u}$ is u-compact.

Proof. By the definition of $S$-spaces, $e-w^{*}=t_{w}=t_{p}$. Further the hypothesis that every $u$-closed and $u$-precompact set is $u$-compact, coupled with $t_{w}=t_{p}$, implies $E_{u}$ is complete by Theorem 10 [3]. Moreover, completeness of $E_{u}$ implies $t_{p}=t_{c}$ on $E^{\prime}{ }_{u}$, and for the same reason $t_{c}$ on $E_{u}^{1}$ is compatible with duality. Hence $e-w^{*}$, being locolly convex and equal to $t_{c}$ is compatible with duality; and therefore, by the Mackey's theorem ([2], Chapitre IV, $\S 2, \mathrm{~N}^{\circ} 3$, Proposition 4), the closed convex sets of $e-w^{*}=t_{c}$ are the same as those of $\sigma\left(E^{\prime}, E\right)$.

Now let $Q$ be a linear almost closed subspace of $E^{\prime}$. But to say that $Q$ is almost closed is the same as saying that $Q$ is $e-w^{*}$-closed. Hence $Q$, being convex (because it is a linear space) and $e-w^{*}$-closed, is also $\sigma\left(E^{\prime}, E\right)$-closed. This proves that $E$ is $B$-complete.

\section{Corollary 2.1. A complete S-space is B-complete.}

Proof. Since, in a complete l.c. space, every closed precompact set is compact, the Corollary follows from Theorem 2.

In the above theorem, though the condition that every closed precompact set in $E$ be compact is sufficient for an $S$-space to be $B$ complete or complete ([3], Theorem 10) it need not be necessary because the $e-w^{*}$-topology on $E^{\prime}$ for $B$-complete l.c. spaces $E$ need not be locally convex. However, the following propositions deal with necessary and sufficient conditions.

Proposition 1. An $S$-space $E$ is complete if and only if every closed precompact set of $E$ is compact.

Proof. If an 1.c. space is complete then the condition is evidently satisfied. On the other hand, the condition implies $E$ is $B$-complete by Theorem 2 and therefore, a fortiori, complete.

Proposition 2. A complete l.c. space $E$ is an $S$-space if and only if $e-w^{*}$, on its dual $E^{\prime}$, is a locally convex topology.

Proof. The fact that $e-w^{*}$ is locally convex for $S$-spaces follows from the definition. On the other hand, if $e-w^{*}$ is locally convex then $e-w^{*}=t_{w}$ on $E^{\prime}$. By the Theorem 10 [3], completeness implies $t_{w}=t_{p}$. 
Combining these two equations we get $e-w^{*}=t_{w}=t_{p}$ which proves that $E$ is an $S$-space.

CoRollary. An LF-space $E$ is B-complete if $e$-w $w^{*}$ on $E^{\prime}$ is locally convex.

Proof. It follows from Proposition 2 because each $L F$-space is complete.

The following theorem is know as the Krein S̆mulian theorem and is known to be true for Banach spaces and more generally for Fréchet spaces. Here we show that it is true for complete $S$-spaces as well.

Theorem 3. Let $E$ be a complete S-space. For a convex set $M^{\prime}$ in $E^{\prime}$ to be $\sigma\left(E^{\prime}, E\right)$-closed it is necessary and sufficient that $M^{\prime} \cap U^{0}$ be $\sigma\left(E^{\prime} E\right)$-closed for each neighborhood $U$ of 0 in $E$.

Proof. The necessary part is obvious because $U^{0}$ is always $\sigma\left(E^{\prime} E\right)$ closed. That it is sufficient follows from the fact that $E$ is a complete $S$-space and hence $e-w^{*}=t_{c}$ on $E$. But $t_{c}$ is compatible with duality (because $E$ is complete) and therefore closed convex sets under $e-w^{*}$ and $\sigma\left(E^{\prime}, E\right)$ are the same by the Mackey's Theorem ([2], Chapitre IV, §2, $\mathrm{N}^{\circ}$ 3, Prop. 4). Now the Theorem follows by observing that saying $M^{\prime} \cap U^{0}$ is $\sigma\left(E^{\prime} E\right)$-closed for each neighborhood $U$ of 0 in $E$ is equivalent to saying that $M^{\prime}$ is $e-w^{*}$-closed.

J. L. Kelley [12] has recently studied hypercomplete spaces. $\mathrm{He}$ proved: A linear topological space $F$ is hypercomplete if and only if each $e-w^{*}$-closed convex circled subset of $F^{\prime}$ is $\sigma\left(E^{\prime}, E\right)$-closed. $\mathrm{He}$ studied hypercomplete spaces in connection with the closed graph Theorem. He does not touch on any of the theorem proved here for $S$-spaces. It is clear from Theorem 3 that a complete $S$-space is hypercomplete. We do not know if the converse is true. We conjecture that complete $S$-spaces and hypercomplete spaces are the same.

\section{Completion of $S$-spaces.}

THEOREM 4. The completion $\hat{E}_{u}$ of an $S$-space $E_{u}$ is an S-space.

Proof. We can identify the duals $E_{u}^{\prime}$ and $\hat{E}_{u}^{\prime}$ of $E_{u}$ and $\hat{E}_{u}$ respectively, under the mapping $i: f \rightarrow \hat{f}\left(f \in E_{u}^{\prime}, \hat{f} \in \hat{E}_{u}^{\prime}\right)$ where $\hat{f}$ is the unique extension of $f$. As $i$ is an algebraic isomorphism of $E_{u}^{\prime}$ onto $\hat{E}_{u}^{\prime}$, it is easy to see that $e-w^{*}$ on $E_{u}^{\prime}$ and $\widehat{e-w^{*}}$ on $\hat{E}_{u}^{\prime}$ are the same. Let $t_{p}$ and $\hat{t}_{p}$ denote the uniform convergence topologies on precompact sets of $E_{u}$ and $\hat{E}_{u}$ respectively. Since every precompact set of $E_{u}$ is precompact 
in $\hat{E}_{u}$, we have $\hat{t}_{p} \supset t_{p}$. But in general $\widehat{e-w^{*}} \supset \hat{t}_{p}$ and so $\widehat{e-w^{*}} \supset \hat{t}_{p} \supset t_{p}$. By hypothesis $E_{u}$ is an $S$-space (i.e., $e-w^{*}=t_{p}$ ) and therefore $e-w^{*}=$ $\widehat{e-w^{*}}=\hat{t}_{p}=t_{p}$ which proves the Theorem.

The following Corollary 4.1 follows immediately from the above 'Theorem.

Corollary 4.1. The completion $\hat{E}_{u}$ of an S-space $E_{u}$ can be $o b$ tained by completing the precompact sets of $E_{u}$ only.

REMARK. The above Corollary shows that $S$-spaces and metrizable 1.c. spaces share the property that their completion can be obtained by completing their precompact sets only. In the case of metrizable spaces, precompact sets are to be replaced by Cauchy sequences.

CoRollary 4.2. If $E_{u}$ is an S-space then $\hat{E}_{u}$ is B-complete.

Proof. By the above theorem, $\hat{E}_{u}$ is an $S$-space, and being complete it is $B$-complete by Corollary 2.1 .

6. S-spaces and bornological spaces. In view of the fact that every metrizable 1.c. space is bornological ([2], Chapitre III, §2, Exercise 15) as well as it is an S-space ([3], Theorem 12), one would like to know if there is any relation between bornological and $S$-spaces. We give an example of bornological l.c. space which is not an $S$-space. Actually we prove more than that. More specifically we have the following:

Theorem 5. A bornological l.c. space need not be an S-space. Also a complete, bornological, $t$-space need not be an $S$-space.

Proof. For this let $E$ be an infinite dimensional Banach space on the field of the reals. It is easy to see that $E_{w}$ is a bornological l.c. space. On the other hand, Collins [3] has shown that $E_{w}$ is not $B$-complete and hence not an $S$-space. For the second part of the theorem, the same example works because $E_{w}$ is a reflexive, complete $t$-space ([2], Chapitre II, §2, Exercise 3, and Chapitre III, \$1, Exercise 3).

7. Subspaces of an $S$-space. In this section we shall show that a subspace of a complete $S$-space with the Closure property (defined below) is an $S$-space.

Definition 2. A TVS $E$ is said to satisfy CP (Closure-property) if for any dense subspace $Q$ of $E$, each precompact set of $E$ is contained in the closure of a precompact set of $Q$. 
Remark. It is clear from the Definition that CP is equivalent to saying that the closure of a dense subspace $Q$ of a TVS $E$ is obtained by taking the closures of its precompact sets only.

In gendral an $S$-space need not satisfy CP. Every metrizable 1.c. space of course does so.

First of all we prove the following:

Proposition 3. A closed subspace $Q$ of a complete $S$-space $E$ is also a complete $S$-space.

Proof. $Q$ is clearly complete and therefore, it is sufficient to show that $e-w^{*}{ }_{1}=t_{c_{1}}$ on $Q^{\prime}=E^{\prime} / Q^{0}$, where $e-w^{*}{ }_{1}$ is the finest topology which coincides with $\sigma\left(Q^{\prime}, Q\right)$ on each equicontinuous set of $Q^{\prime}$, and $t_{c_{1}}$ is the uniform convergence topology on convex compact sets of $Q$. By Exercise 12(c) ([2], Chapitre IV, $\left.\S 3, \mathrm{~N}^{\circ} 4\right)$ the quotient topology $t_{c}{ }^{q}$ on $Q^{\prime}$ is the same as $t_{c_{1}}$ i.e., $t_{c}^{q}=t_{c_{1}}$ because the convex hull of a compact set in $E$ as well as in $Q$, is compact because of $E$ and $Q$ being complete. But the equality $e-w^{*}=t_{c}$ on $E^{\prime}$ (because $E$ is a complete $S$-space) implies $e-w^{* q}=t_{c}^{q}$ on $Q^{\prime}$ (where $e-w^{* q}$ is the quotient topology of $e-w^{*}$ on $Q^{\prime}$ and clearly Hausdorff because $Q^{0}$ is weakly closed and therefore $e-w^{*}$-closed due to continuity of the identity mapping). Hence we have

$$
e-w^{* q}=t_{c_{1}}
$$

Furthermore, $e-w^{* q}$ coincides with $\sigma\left(Q^{\prime} Q\right)$ on each equicontinuous set of $Q^{\prime}$ because $t_{c_{1}}=t_{p_{1}}$ does so according to Proposition 5 ([2], Chapitre III, $\left.\S 3, N^{\circ} 5\right)$. Since $e-w^{*}{ }_{1}$ is the finest topology which coincides. with $\sigma\left(Q^{\prime}, Q\right)$ on each equicontinuous set of $Q^{\prime}$, we have

$$
e-w_{1}^{*} \supset e-w^{* q} \text {. }
$$

In view of (i) it is clearly sufficient to show the reverse inclusion in order to complete the proof. For this let $W^{\prime}$ be an $e-w^{*}{ }_{1}$-open neighborhood of 0 in $Q^{\prime}$. We wish to show that $\phi^{-1}\left(W^{\prime}\right)$, where $\phi$ is the canonical mapping: $E^{\prime} \rightarrow E^{\prime} / Q^{0}$, is an $e-w^{*}$-open neighborhood of 0 in $E^{\prime}$. For each neighborhood $U$ of 0 in $E, U^{0}$ is $\sigma\left(E^{\prime}, E\right)$-compact in $E^{\prime}$. Let $\phi\left(U^{0}\right)=Y$ and $\phi^{-1}(Y)=X$. Due to $\sigma\left(E^{\prime}, E\right)$-continuity of $\phi$, $Y$ is $\sigma\left(E^{\prime}, E\right)$-compact and $X$ is $\sigma\left(E^{\prime}, E\right)$-closed in $E^{\prime}$. On the compact, set $Y, e-w^{*}{ }_{1}$ and $\sigma\left(Q^{\prime}, Q\right)$ are the same. Let us now consider

$$
\phi^{-1}\left(W^{\prime}\right) \cap X .
$$

Clearly

$$
\phi^{-1}\left(W^{\prime}\right) \cap X=\phi^{-1}\left(W^{\prime} \cap Y\right) .
$$

Since $W^{\prime}$ is an $e-w^{*}{ }_{1}$-open neighborhood and $Y$ is compact, $W^{\prime} \cap Y$ is: 
relatively weakly open in $X$. Further, since $U^{0}$ is $\sigma\left(E^{\prime}, E\right)$-closed and $\sigma\left(E^{\prime}, E\right)$-compact,

$$
\phi^{-1}\left(W^{\prime}\right) \cap X \cap U^{0}=\phi^{-1}\left(W^{\prime}\right) \cap U^{0}
$$

(because $\left.X \supset U^{0}\right)$ is $\sigma\left(E^{\prime}, E\right)$-open for each neighborhood $U$ of 0 in $E$. This shows, by definition, that $\phi^{-1}\left(W^{\prime}\right)$ is an $e$ - $w^{*}$-open neighborhood of 0 in $E^{\prime}$ and therefore $e-w_{1}^{*} \subset e-w^{* q}$. This completes the proof.

Proposition 4. Let $E$ be an $S$-space satisfying CP. Then every dense subspace $Q$ of $E$ is an $S$-space.

Proof. Density of $Q$ implies $Q^{\prime}=E^{\prime}$. Since for every neighborhood $U$ of 0 in $Q, U^{0}=(\bar{U})^{0}$ (where $\bar{U}$ is the closure of $U$ in $E$ and hence a neighborhood of 0 in $E$ ), the topologies $e-w^{*}$ and $e$-w $w_{1}^{*}$ with regard to $E$ and $Q$ respectively are the same on $E^{\prime}$. By hypothesis for each precompact set $P$ in $E$, there exists a precompact set $P_{1}$ in $Q$ such that $\bar{P}_{1} \supset P$. This means that $t_{p_{1}}=t_{p}$ on $E^{\prime}$ (where $t_{p_{1}}$ is the uniform convergence topology over precompact sets of $Q$ ) and therefore,

$$
e-w^{*}=e-w_{1}^{*} \supset t_{p_{1}}=t_{p} .
$$

But $E$ is an $S$-space and therefore $e-w_{1}^{*}=t_{p_{1}}$. This proves the proposition.

THeORem 6. Let $E$ be a complete S-space satisfying CP. Then every subspace $Q$ of $E$ is an $S$-space.

Proof. According to Proposition $3, \bar{Q}$ is an $S$-space in which $Q$ is a dense subspace and satisfies CP. Therefore, by Proposition $4, Q$ is an S-space.

\section{Permanence property of $S$-spaces.}

THeOREM 7. Let $f$ be a linear, continuous and almost open mapping of an l.c. space $E$ onto another l.c. space $F$. If $E$ is an S-space then $F$ is also an S-space.

Proof. By hypothesis, the transpose mapping $f^{\prime}: F^{\prime} \rightarrow E^{\prime}$ is a homeomorphism (into). Therefore $F^{\prime}$ can be identified with its image $f^{\prime}\left(F^{\prime}\right)$ in $E^{\prime}$. Let $W^{\prime}$ be an $e-w^{*}$-open neighborhood of 0 in $F^{\prime}$ i.e., $W^{\prime} \cap V^{0}$ is $\sigma\left(F^{\prime}, F\right)$-open for each neighborhood $V$ of 0 in $F$. Since

$$
\begin{aligned}
W^{\prime} \cap U^{0} & =W^{\prime} \cap f^{\prime}\left(f^{\prime-1}\left(U^{0}\right)\right)=W^{\prime} \cap f^{\prime}(f(U))^{0} \\
& =W^{\prime} \cap f^{\prime}(\overline{f(U)})^{0}=W^{\prime} \cap f^{\prime}\left(V^{0}\right) \\
& =W^{\prime} \cap V^{0}
\end{aligned}
$$


is $\sigma\left(F^{\prime}, F\right)$-open for each neighborhood $U$ of 0 in $E$ (bacause $f$ beingalmost open implies $\overline{f(U)}=V$ ), and therefore $\sigma\left(E^{\prime}, E\right)$-open, $W^{\prime}$ is an $e-w^{*}$-open neighborhood of 0 in $E^{\prime} . \quad E$ being an $S$-space, due to Theorem 1 there exists a precompact set $P$ in $E$ such that $P^{0} \subset W^{\prime}$. But

$$
(f(P))^{0}=f^{\prime-1}\left(P^{0}\right) \subset f^{\prime-1}\left(W^{\prime}\right)=W^{\prime}
$$

(because $f^{\prime}$ is $1: 1$ ) implies that $F$ is an $S$-space due to Theorem 1 , because $f$ being continuous and $P$ a precompact set implies $f(P)$ is. precompact in $F$.

CoRollary 7.1. Let $E$ and $F$ be l.c. spaces and $f$ a linear, continuous and open mapping of $E$ onto $F$. Then $F$ is an $S$-space if $E$ is an S-space.

Proof. The Corollary is immediate from Theorem 7 because every open mapping is almost open.

CoRollary 7.2. Let $E$ be an S-space and $M$ a closed subspace of $E$. Then $E / M$ is an S-space.

COROLLARY 7.3. Let $E_{u}$ be an l.c. space and $v$ another locally convex topology on $E$ such that $u \supset v$ and $v(u)=v$. Then $E_{v}$ is an $S$-space if $E_{u}$ is an S-space.

COROLlaRY 7.4. If every bornological space is an S-space then every quasibarrelled space is also an S-space.

Proof. For this let $E_{u}$ be a quasi-barrelled space and $E_{B}$ its associated bornological space ([2], Chap. III, p. 13). By Theorem 2.3 [17], the identity mapping: $E_{B} \rightarrow E_{u}$ is linear, continuous and almost open. Hence the Corollary follows from Theorem 7 .

REMARK. Corollary 7.4 can also be taken to prove the first part of Theorem 5 .

9. Strict inductive limit of $S$-spaces. From a result $\left([9], 5^{\circ}\right.$, p. 93$)$ due to Grothendieck it follows that an $L F$-space need not be $B$-complete and hence the strict inductive limit of $S$-spaces need not be an $S$-space. In Proposition 2 we have had a sufficient condition for any complete l.c. space, in particular, for any $L F$-space to be an $S$-space. That condition is too obvious and almost the definition of an $S$-space. Here we shall have another condition which is more practicable.

First of all we have some lemmas. 
Lemma 1. Let $\mathfrak{S}_{1}$ and $\mathfrak{\Im}_{2}$ be two collections of convex compact sets of an l.c. space $E_{u}$ such that $\mathfrak{F}_{1} \subset \mathfrak{F}_{2}$. Let $k\left(\mathfrak{F}_{1}, u\right)$ and $k\left(\mathfrak{F}_{2}, u\right)$ denote the finest topologies which coincide with $u$ on each $C_{1} \in \mathbb{S}_{1}$ and $C_{2} \in \mathfrak{G}_{2}$ respectively. Then $k\left(\mathfrak{S}_{1}, u\right) \supset k\left(\mathfrak{E}_{2}, u\right)$.

Proof. Let $W$ be an open set in $k\left(\mathfrak{F}_{2}, u\right)$ i.e., $W \cap C_{2}$ is open under the induced topology $u$ on $C_{2}$ for each $C_{2} \in \mathfrak{S}_{2}$. Since each $C_{1} \in \mathfrak{\Im}_{2}$, therefore $W \cap C_{1}$ is open under the induced $u$-topology on $C_{1}$ for each $C_{1} \in \mathfrak{\Im}_{1}$ and hence $W$ is an open set in $k\left(\mathfrak{\Im}_{1}, u\right)$.

Lemma 2. Let $E$ be an l.c. space and $E^{\prime}$ its dual. Let $\mathfrak{S}_{1}$ and $\widetilde{F}_{2}$ be two collections of all convex $t_{p}$-compact and convex closed equicontinuous sets of $E^{\prime}$ respectively. Let $k\left(\mathfrak{\complement}_{1}, t_{p}\right)$ and $e-w^{*}$ be the topologies defined as usual. Then $k\left(\mathfrak{S}_{1}, t_{p}\right) \supset e-w^{*} \supset t_{p}$.

Proof. Since each $t_{p}$-compact set is $\sigma$-compact because $t_{p} \supset \sigma\left(E^{\prime}, E\right)$, and $t_{p}$ coincides with $\sigma\left(E^{\prime}, E\right)$ on each equicontinuous set, the first inclusion in the Lemma follows from Lemma 1. The second inclusion is true in general.

Lemma 3. Let $E_{u}$ be an l.c. space which has a countable fundamental system of u-precompact sets. Then $E$ is an $S$-space.

Proof. In view of Lemma 2 it is sufficient to prove that $k\left(\mathfrak{S}_{1}, t_{p}\right)=t_{p}$ on $E^{\prime}$. But this follows from the fact that, by hypothesis, $t_{p}$ is metrizable and therefore, $k\left(\varsigma_{1}, t_{p}\right)$ being its $k$-extension is equal to $t_{p}$ ([13], p. 231, Theorem 13).

THeORem 8. Let $E_{u}$ be a complete l.c. space which has a countable fundamental system of u-precompact sets. Then $E_{u}$ is B-complete.

Proof. By Lemma 3, $E$ is an $S$-space and being complete it is a complete $S$-space. Therefore, by Corollary $2.1, E_{u}$ is $B$-complete.

THEOREM 9. Let $E$ be the strict inductive limit of complete l.c. spaces $E_{n}$ 's $(n \geqq 1)$ such that each $E_{n}$ contains a countable fundamental system of precompact sets. Then $E$ is a complete $S$-space and a fortiori B-complete.

Proof. By Exercise 9 ([2], Chapitre II, $\left.\S 2, \mathrm{~N}^{\circ} 5\right) E$ is complete. Moreover, each precompact set of $E$ is contained in some $E_{m}$ for some $m$ and is a precompact set in $E_{m}$ ([2], Chapitre III, $\S 2, \mathrm{~N}^{\circ} 4$, Proposition 6). Since each $E_{m}$ contains only a countable fundamental system 
of precompact sets, so does $E$. Hence by Theorem $8, E$ is $B$-complete.

Corollary 9.1. An LF-space $E$ with a defining sequence of Fréchet spaces $E_{n}$ 's is B-complete provided each $E_{n}$ has a countable fundamental system of precompact sets.

CoRollary 9.2. Let $E_{n}(n \geqq 1)$ be a sequence of complete l.c. spaces each of which contains a countable fundamental system of precompact sets. Let $E$ be the direct sum of $E_{n}$ 's. Then $E$ is acompl ete $S$-space and a fortiori B-complete.

REMARK. The $t$-spaces and quasi-barrelled spaces with a countable fundamental system of compact sets have been studied by Dieudonné [7], and by Mahowald and Gould [17] respectively.

10. Dual of an $S$-space. It is known ([19], 6.5) that the dual $E^{\prime}$, endowed with the $t_{c}$-topology of a Fréchet spach $E$ is $B$-complete for all locally convex topologies finer than $t_{c}$ and coarser the $\tau\left(E^{\prime}, E\right)$. However, this need not be the case for $B$-complete l.c. spaces is shown as follows:

Proposition 5. The dual of a $B$-complete l.c. space, endowed with the $t_{c}$-topology, need not be $B$-complete.

Proof. Consider $E=I_{\alpha} R_{\alpha}$ (an arbitrary Cartesion product of real numbers), endowed with the Cartesian product topology. According to Corollary 17.2 [3], $E$ is $B$-complete and every closed bounded set in $E$ is compact ([11], Lemma 6). Hence $t_{c}=\beta$ on $E^{\prime}$. As $\beta$ is the direct sum topology of finite-dimensional spaces, it is the finest locally convex topology and $E^{\prime \beta}=E^{\prime t_{c}}$ is a complete, bornological and reflexive $t$-space. We show that it is not $B$-complete.

For this let $F$ be an infinite dimensional Banach space. It is clear that the identity mapping $i: F_{w} \rightarrow F$ is linear, continuous, $1: 1$ and onto. It is also known ([3], pp. 271-272) that $i$ is almost open but not open. Hence $F^{\prime}$ is dense and almost closed in $F_{w}^{\prime}$ but not closed. This shows that $F_{w}$ is not $B_{r}$-complete (a fortiori not $B$-complete). But then it is known that $F_{w}$ is nothing more than $\sum_{\alpha \in H} R_{\alpha}$, where $H$ is the Hamel base and $R_{\alpha}$ is a copy of real numbers, and therefore $F^{\prime}=\sum_{\alpha \in B} R_{\alpha}$. Thus, if we take $F_{w}$ for $E^{t_{c}}=E^{\prime \beta}$ in the Proposition, the conclusion immediately follows.

THEOREM 10. Let $E_{u}$ be a complete S-space satisfying CP. Then $E^{\prime t_{c}}$ is $B_{r}$-complete for all locally convex topologies finer that $t_{c}$ and 
coarser that $\tau\left(E^{\prime}, E\right)$.

Proof. Let $Q$ be an almost closed (i.e., $Q \cap C^{00}$ is $\sigma\left(E, E^{\prime}\right)$-closed for each $u$-compact set $C$ of $E$ ) dense subspace of $E$. By Proposition $4, Q$ is an $S$-space. Further, the density of $Q$ implies $E^{\prime}=Q^{\prime}$ and hence the $e-w^{*}$-topology is the same with respect to $E$ and $Q$. $Q$ being an $S$-space, $e-w^{*}$ is locally convex and $e-w^{*}=t_{p_{1}}$, where $t_{p_{1}}$ is the uniform convergence topology over precompact sets of $Q$ on $E^{\prime}$.

Now we show that the almost closedness of $Q$ implies every closed precompact set in $Q$ is compact. For this let $t_{c}$ and $t_{c_{1}}$, on $E^{\prime}$, be the uniform convergence topologies over convex compact sets of $E$ and $Q$ respectively. Since $E$ is complete, it is clear that $t_{c}$ and $t_{c_{1}}$ are compatible with duality. It is also clear that $t_{c_{1}} \subset t_{c}$. By virtue of result 3.2 [19], almost closedness of $Q$ in $E$ is equivalent to the fact that the identity mapping: $E^{\prime t_{c}} \rightarrow E^{\prime t_{c_{1}}}$ is almost open or in Pták's notation:

$$
t_{c_{1}}\left(t_{c}\right)=t_{c_{1}} \text {. }
$$

But in general ([19], Theorem 5.10) or ([3], Theorem 10)

$$
t_{c_{1}}\left(t_{w_{1}}\right)=t_{p_{1}}
$$

where $t_{w_{1}}$ is the finest locally convex topology which coincides with $\sigma\left(Q^{\prime}, Q\right)$ on each equicontinuous set of $Q^{\prime}$. But since $Q$ is an $S$-space, so

$$
t_{c_{1}}\left(e-w^{*}\right)=t_{p_{1}} .
$$

But $E$ being a complete $S$-space implies

$$
e-w^{*}=t_{p}=t_{c} .
$$

Hence we have

$$
t_{c_{1}}=t_{c_{1}}\left(t_{c}\right)=t_{c_{1}}\left(e-w^{*}\right)=t_{p_{1}}
$$

which proves that closed precompact sets in $Q$ are compact. By Proposition 1, this implies that $Q$ is complete and hence closed in $E$. This shows that $Q=E$ because $Q$ is dense in $E$. This proves the first part of the Theorem. The remainder follows from Pták's result 4.3 [19].

11. S-spaces which are strong duals of certain 1.c. spaces.

THEOREm 11. The strong dual of a semi-reflexive $t$-space $E_{u}$ is a complete S-space and hence a fortiori B-complete.

Proof. It is sufficient to prove that $E^{\prime \beta}$ is an $S$-space because, according to Exercise 3(c) ([2], Chapitre IV, §3, $\mathrm{N}^{\circ} 4$ ) coupled with the observation that a metrizable l.c. space is bornological, $E^{\prime \beta}$ is complete. 
Furthermore, semi-reflexiveness implies that $E^{\prime \beta \prime}=E$ and $E^{\prime \beta}$ is a $t$-space ([2], Chapitre IV, $\S 3, \mathrm{~N}^{\circ} 3$, Proposition 4); and also that $\beta=\tau\left(E^{\prime}, E\right)$. Further, according to Exercise 12(b) ([2], Chapitre IV, $\S 1, \mathrm{~N}^{\circ} 6$ ) every $\sigma$-bounded set (hence every $\tau$-bounded set because $\sigma$-bounded and $\tau$-bounded sets are the same by the Mackey's Theorem) is $\tau$-precompact. But every $\tau$-precompact set, being $\sigma$-bounded, is equicontinuous because $E$ is a $t$-space by hypothesis. Hence $u=t_{p}$ on $E$, where $t_{p}$ is the uniform convergence topology over $\tau$-precompact sets of $E^{\prime}$. This proves that $u$ coincides with $\sigma\left(E, E^{\prime}\right)$ on each equicontinuous set of $E$ because $t_{p}$ does so. But $t_{p}=u$ being metrizable, $e-w^{*}=t_{p}$ because of Theorem 13 ([13], p. 231) and the argument used in Lemma 3 . Hence $E^{\prime \beta}$ is a complete $S$-space and by Corollary 2.1 , it is $B$-complete.

COROLlaRY 11.1 The strong dual of a semi-reflexive Fréchet space is a complete $S$-space and a fortiori B-complete.

Proof. The Corollary follows from Theorem 11 because every Fréchet space is metrizable and a $t$-space.

COROLlaRY 11.2 The strong dual of a metrizable Montel space is a complete S-space.

Proof. The Corollary follows from Theorem 11 becanse a Montel space is reflexive (hence semi-reflexive) and is a $t$-space.

The following Corollaries are immediate from the above Theorem and $\$ 9$. Their proofs are omitted.

COROLLARY 11.3 The strong dual of a Montel space $E_{u}$ is a complete $S$-space and hence B-complete provided $E^{\beta}$ is metrizable.

CoRollaRY 11.4 The strong dual of a Montel space $E_{u}$ is a complete S-space provided $E_{u}$ contains a countable fundamental system of bounded sets.

CoRollaRY 11.5 A Montel space $E$ is a complete S-space provided $E$ contains a countable fundamental system of bounded sets.

REMARK. In view of the fact that every metrizable l.c. space is bornological, one might be tempted to prove Theorem 11 for semireflexive bornological $t$-spaces. But this is not true due to an example given in Theorem 5 .

12. the open mapping theorem. Banach [1] proved that if $E$ and $F$ are two $F$-spaces then a linear and continuous mapping of $E$ 
onto $F$ is open. Our object is to replace the range space $F$ in Banach's theorem by a more general linear space which we call $T$-spaces (defined below). The purpose of defining $T$-spaces is to knock out local convexity from $t$-spaces. For locally convex linear spaces the Theorem has been carried over to more general linear spaces Fréchet space, for example, see Dieudonné et Schwartz [6], Grothendieck [10], Pták [19], Robertsons [21].

Definition 3. A topological vector space is called a $T$-space if every closed, circled and absorbing set is a neighborhood of 0 .

Theorem 12. If $E$ is an $F$-space and $F$ a T-space then a linear and continuous mapping $f$ of $E$ onto $F$ is open.

Since $E$ is an $F$-space, there exists a countable fundamental system of neighborhoods $U_{n}(n \geqq 1)$ of 0 in $E$ such that $U_{n}$ 's are closed, circled and absorbing with the following properties:

(a) $U_{n-1}+U_{n+1} \subset U_{n}$ for all $n \geqq 1$,

(b) $\bigcap_{n=1}^{\infty} U_{n}=\{0\}$.

For the proof of theorem we need the following two lemmas. The proof of Lemma 4 is straightforward and therefore omitted.

LEMMa 4. Let $E$ be a topological vector space and $F$ a T-space. Let $f$ be a linear mapping of $E$ onto $F$. Then for each neighborhood $U$ of 0 in $E, \overline{f(U)}$ is a neighborhood of 0 in $F$.

Now for each neighborhood $U_{n}$ of 0 in $E$, let us define

$$
\overline{f\left(U_{n}\right)}=W_{n} \text { for each } n \text {. }
$$

Evidently $W_{n \div 1} \subset W_{n}$ for all $n \geqq 1$.

LEMMA 5.

$$
\bigcap_{n=1}^{\infty} W_{n}=\{0\} .
$$

Proof. Let $y \in \bigcap_{n=1}^{\infty} W_{n}$. Then $y \in \overline{f\left(U_{n}\right)}$ for all $n \geqq 1$. Let $V$ be a closed and circled neighborhood of 0 in $F$, then $(y+V) \cap f\left(U_{n}\right) \neq \phi$ for all $n \geqq 1$. That means there exists $x_{n} \in U_{n}$ such that $f\left(x_{n}\right) \in y+V$ for all $n \geqq 1$, or

$$
f\left(x_{n}\right)-y \in V
$$


since $x_{n} \in U_{n}$ and $U_{n}(n \geqq 1)$ forms a decreassing sequence of a fundamental system of neighborhoods of 0 in $E, x_{n} \rightarrow 0$. $f$ being continuous and $V$ being closed, (i) implies $f(0)-y \in V$ or $y \in V$. But $V$ is arbitrary, therefore $y=0$.

Proof of Theorem 12. Since $U_{n}(n \geqq 1)$ forms a fundamental system of neighborhoods, so in order to prove that $f$ is open, i.e., for any open set $P$ in $E f(P)$ is open in $F$, it is sufficient to show that $f\left(U_{n}\right)$ is a neighborhood of 0 in $F$ for each $n$. More specifically what we shall show is that $f\left(U_{k}\right) \supset W_{k+1}$ for any $k$.

Let $y \in W_{k+1}$. Since $f\left(U_{k+1}\right)$ is dense in $W_{k+1}$, there exists $y_{1}$ with a preimage $x_{1}=f^{-1}\left(y_{1}\right) \in U_{k+1}$ such that $y-y_{1} \in W_{k+2}$. Inductively proceeding we assume that there exists $y_{n}$ with a preimage $x_{n}=f^{-1}\left(y_{n}\right) \in U_{k+n}$ such that

$$
y-\sum_{j=1}^{n} y_{j} \in W_{k+n+1}
$$

Further, since

$$
x_{n}+x_{n+1}+\cdots+x_{n+p} \in U_{k+n}+\cdots+U_{k+n+p}
$$

and

$$
U_{k+n} \supset U_{k+n+1}+\cdots+U_{k+n+n}
$$

therefore

$$
x_{n}+x_{n+1}+\cdots+x_{n+p} \in U_{k+n}+U_{k+n} \subset U_{k+n-1} .
$$

Now given any arbitrary neighborhood $V$ of 0 , we can choose $N$ large enough so that $U_{k+n-1} \subset V$ for $n \geqq N$ and hence

$$
x_{n}+\cdots+x_{n+p} \in V \text { for all } n \geqq N \text { and } p \geqq 0 \text {. }
$$

This shows that the partial sums of the series $\sum_{j=1}^{\infty} x_{j}$ form a Cauchy sequence. But $E$ is complete, therefore there exists $x \in E$ such that

$$
x=\sum_{j=1}^{\infty} x_{j} .
$$

By putting $n=1$ in (B) we have

$$
\sum_{j=1}^{m} x_{j} \in U_{k} \text { for each } m,
$$

and since $U_{k}$ is closed so (letting $m \rightarrow \infty$ ),

$$
x=\sum_{j=1}^{\infty} x_{j} \in U_{k} .
$$


$f$ being continuous implies

$$
f(x)=\sum_{j=1}^{\infty} f\left(x_{\jmath}\right)=\sum_{j=1}^{\infty} y_{j} .
$$

From (A) follows that

$$
y-\sum_{j=1}^{n+p} y_{j} \in W_{k+n+p+1} \subset W_{k+n ! 1} \text { for } p \geqq 0 .
$$

Since $p$ can be chosen arbitrarily, so

$$
y-\sum_{j=1}^{m} y_{j} \in W_{k+n+1} \text { for large } m,
$$

and $W_{k+n+1}$ being closed (letting $m \rightarrow \infty$ ) we have

$$
y-\sum_{\jmath=1}^{\infty} y_{j} \in W_{k+n+1}
$$

or from (D), $y-f(x) \in W_{k+n+1}$ for each $n$. That implies

$$
y-f(x) \in \bigcap_{n=1}^{\infty} W_{k+n+1} .
$$

Hence by Lemma 5, $y=f(x)$. But since, from (C) $x \in U_{k}$, therefore

$$
y=f(x) \in f\left(U_{k}\right)
$$

or $f\left(U_{k}\right) \supset W_{k+1}$. This proves the theorem.

Theorem 13. Let $E$ be a Fréchet space and $F$ an l.c. space. Let $f$ be a linear, continuous and almost open mapping of $E$ into $F$. Then $\overline{f(E)}$ is a t-space.

Proof. It is clear that $\overline{f(E)}$ is a closed linear subspace of $F$. Let $B$ be a barrel in $\overline{f(E)}$, then $B$ is closed in $F$ as well, and $f$ being linear and continuous implies $f^{-1}(B)$ is closed, convex, circled and absorbing in $E$. But $E$ being a Fréchet space and therefore a $t$-space ([2], Chapitre III, $\S 1$, Corollary of Proposition 1) implies $f^{-1}(B)$ is a neighborhood of 0 in $E$. Since $f$ is almost open, $\overline{f\left(f^{-1}(B)\right)}=\bar{B}=B$ is a neighborhood of 0 and hence $\overline{f(E)}$ is a $t$-space.

By virtue of the facts that every complete $S$-space and the completion of an $S$-space are $B$-complete the following Theorems 14 and 15 are just particular cases of Robertsons' Theorems 3 and 4 [21], and of Robertson's Theorems 6 and 7 [22].

Theorem 14. Let $E$ be a complete $S$-space and $F$ a $t$-space. Then: (a) A linear and continuous mapping $f$ of $E$ onto $F$ is open. 
(b) A linear mapping $g$ of $F$ into $E$ with the closed graph is continuous.

THEOREM 15. Let $E$ be an S-space and $F$ a $t$-space. Then:

(a) A linear and continuous mapping $f$ of $E$ onto $F$ is open provided $f$ satisfies the filter condition ([22], Theorem 6).

(b) A linear mapping $g$ of $F$ into $E$ with the closed graph is continuous provided $g$ satisfies the inverse filter condition ([22], Theorem 7).

We have also investigated a kind of "converse" of the open mapping theorem. More precisely, let $\mathfrak{F}$ (or $\mathfrak{I}$ ) be the class of all Fréchet spaces (or $t$-spaces) $F$. We ask if a locally convex linear space $E$ for which a linear, continuous $1: 1$ and almost open mapping $f$ of $E$ onto $F$ (for each $F \in \mathfrak{F}$ or $\mathfrak{I}$ ) is actually open, is indeed a Fréchet space (or a $B$-complete l.c. space). We have answered both of these questions in the negative and will be published later on.

\section{BIBLIOGRAPHY}

1. S. Banach, Théorie des Opérations Linéares, Warsaw, 1932.

2. N. Bourbaki, Éléments de Mathématique, Espaces vectoriels topologiques, Chapitre I-V livre V, Paris, 1953, 1955.

3. H. S. Collins Completeness and compactness in linear topological spaces, Trans. Amer. Math. Soc., 79 (1955), 256-280.

4. J. Dieudonné, Natural Homomorphisms in Banach Spaces, Proc. Amer. Math. Soc. 1 (1950), 54-59.

5. Recent developments in the theory of locally convex vector spaces, Bull. Amer. Math. Soc., 59 (1953), 495-512.

6. , et L. Schwartz, La dualite dans les espaces $(F)$ et $(L F)$, Ann. Inst. Fourier, Grenoble (1950), 61-101.

7. __ Denumerability conditions in locally convex vector spaces, Proc. Amer. Math. Soc. 8 (1957), 367-372.

8. A. Grothendieck, Sur la Completion du dual d'un espace vectoriel localement convexe, C. R. Acad. Sc. t. 230 (1950), 605-606.

9. _L Sur les espaces $(F)$ et $(D F)$, Summa Bras. Math. t. 3 (1954), 57-123.

10. (_ Produits tensoriels topologique et espaces nucléaires, Mem. Amer. Math. Soc.,

16 (1955).

11. S. Kaplan, Cartesian products of reals, Amer. J. Math., 74 (1952), 936-954.

12. J. L. Kelley, Hypercomplete linear topological spaces, Michigan Math. J.. 5 (1958), $235-246$.

13. __ General Topology, New York Van Nostrand, 1955.

14. A. Kolmogoroff, Zur Normierbarkeit eines allgemeinen topologischen linearen Raumes, Studia Math., 5 (1935), 29-33.

15. G. Köthe, Über zwei Sätze von Banach, Math. Zeitschrift 53 (1950-51), 203-209.

16. G. Mackey, On infinite dimensional linear spaces, Trans. Amer. Math. Soc., 57 (1945), 155-207.

17. M. Mahowald and G. Gould, Quasi-barrelled locally convex spaces, Proc., Amer. Math. Soc., 11 (1960), 811-816.

18. V. Pták, On complete topological linear spacis, Cechoslovack Math. J. 3 (78) 285-290 
and 301-364; (1953) (Russian and English Summary).

19. , Completeness and the open-mapping theorem, Bull. Scoiete Math. de France t. 86 (1958), 41-74.

20. G. T. Roberts, The bounded weak topology and completeness in vector spaces, Proc. Camb. Phil. Soc., 49 (1953), 183-189.

21. A. P. Robertson and W. Robertson, On the closed graph theorem, Proc. Glasgow Math. Ass. 3 (1956), 9-12.

22. W. Robertson, Completion of topological vector spaces, Proc. London Math. Soc. 8 (1958), 242-257.

SYRACUSE UNIVERSITY 



\section{PACIFIC JOURNAL OF MATHEMATICS}

\section{EDITORS}

Ralph S. Phillips

Stanford University

Stanford, California

M. G. Arsove

University of Washington

Seattle 5, Washington
A. L. Whiteman

University of Southern Californla

Los Angeles 7, California

Lowell J. Paige

University of California

Los Angeles 24, California

\section{ASSOCIATE EDITORS}

E. F. BECKENBACH

D. DERRY

H. L. ROYDEN

E. G. STRAUS

T. M. CHERRY

M. OHTSUKA

E. SPANIER

F. WOLF

\section{SUPPORTING INSTITUTIONS}

UNIVERSITY OF BRITISH COLUMBIA

STANFORD UNIVERSITY

CALIFORNIA INSTITUTE OF TECHNOLOGY

UNIVERSITY OF CALIFORNIA

MONTANA STATE UNIVERSITY

UNIVERSITY OF TOKYO

UNIVERSITY OF UTAH

UNIVERSITY OF NEVADA

NEW MEXICO STATE UNIVERSITY

OREGON STATE UNIVERSITY

UNIVERSITY OF OREGON

OSAKA UNIVERSITY

WASHINGTON STATE UNIVERSITY

UNIVERSITY OF WASHINGTON

UNIVERSITY OF SOUTHERN CALIFORNIA

AMERICAN MATHEMATICAL SOCIETY CALIFORNIA RESEARCH CORPORATION SPACE TECHNOLOGY LABORATORIES NAVAL ORDNANCE TEST STATION 


\section{Pacific Journal of Mathematics}

\section{Vol. 12, No. $1 \quad$ January, 1962}

Jonathan L. Alperin, Groups with finitely many automorphisms $\ldots \ldots \ldots \ldots \ldots \ldots \ldots \ldots$

Martin Arthur Arkowitz, The generalized Whitehead product ................ 7

John D. Baum, Instability and asymptoticity in toplogical dynamics . . . . . . . . . . 25

William Aaron Beyer, Hausdorff dimension of level sets of some Rademacher series .... $\quad 35$

Frank Herbert Brownell, III, A note on Cook's wave-matrix theorem . . . . . . . . . . . . . 47

Gulbank D. Chakerian, An inequality for closed space curves ................. 53

Inge Futtrup Christensen, Some further extensions of a theorem of Marcinkiewicz ....... 59

Charles Vernon Coffman, Linear differential equations on cones in Banach spaces . . . . . 69

Eckford Cohen, Arithmetical notes. III. Certain equally distributed sets of integers . . . . . 77

John Irving Derr and Angus E. Taylor, Operators of meromorphic type with multiple poles

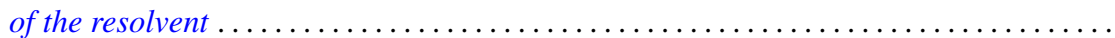

Jacob Feldman, On measurability of stochastic processes in products space .............

Robert S. Freeman, Closed extensions of the Laplace operator determined by a general class of boundary conditions, for unbounded regions ......................

Robert E. Fullerton, Geometric structure of absolute basis systems in a linear topological

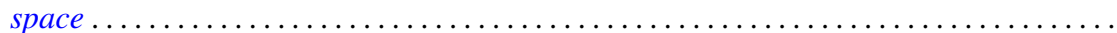

Dieter Gaier, On conformal mapping of nearly circular regions

Andrew Mattei Gleason and Hassler Whitney, The extension of linear functionals defined

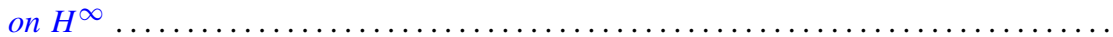

Seymour Goldberg, Closed linear operators and associated continuous linear

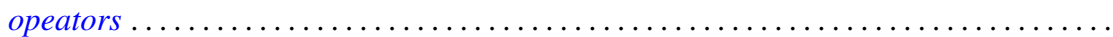

Basil Gordon, Aviezri Siegmund Fraenkel and Ernst Gabor Straus, On the determination of sets by the sets of sums of a certain order

Branko Grünbaum, The dimension of intersections of convex sets. .

Paul Daniel Hill, On the number of pure subgroups

Robert Peter Holten, Generalized Goursat problem . .

Alfred Horn, Eigenvalues of sums of Hermitian matrices ...........

Henry C. Howard, Oscillation and nonoscillation criteria for

$$
y^{\prime \prime}(x)+f(y(x)) p(x)=0
$$

Taqdir Husain, $S$-spaces and the open mapping theorem ...

Richard Eugene Isaac, Markov processes and unique stationary probability measures ...

John Rolfe Isbell, Supercomplete spaces ....................

John Rolfe Isbell, On finite-dimensional uniform spaces. II .........

N. Jacobson, A note on automorphisms of Lie algebras ..............

Antoni A. Kosinski, A theorem on families of acyclic sets and its applications

Marvin David Marcus and H. Minc, The invariance of symmetric functions of singular values...

Ralph David McWilliams, A note on weak sequential convergence.

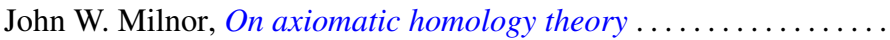

Victor Julius Mizel and Malempati Madhusudana Rao, Nonsymmetric projections in

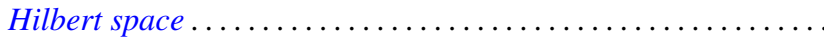

Calvin Cooper Moore, On the Frobenius reciprocity theorem for locally compact

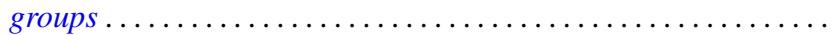

Donald J. Newman, The Gibbs phenomenon for Hausdorff means . 